\title{
ÉTICA Y MEDICINA: LA EXPERIENCIA DEL COLEGIO MÉDICO DEL PERÚ
}

\begin{abstract}
Alfonso Mendoza F1,2,a
\section{RESUMEN}

En este artículo se muestra la labor del Colegio Médico del Perú en lo referente a los procedimientos ético disciplinarios, pero también cómo, más allá del control ético, la Orden ha promovido (vía un conjunto de acciones) una atención de salud técnicamente competente a la vez que respetuosa de la dignidad y de los derechos fundamentales de la persona. De ese conjunto forman parte el establecimiento del Día del Paciente, el énfasis en el mejoramiento de la calidad y la seguridad en la atención de los usuarios de los servicios de salud, la iniciativa para prevenir y tratar los eventos adversos, la regulación y perfeccionamiento de la formación del médico tanto en el pre como en el posgrado, y la demanda por la instauración de un óptimo sistema de salud y de políticas que hagan realidad el derecho a la salud en el marco de los principios de la Bioética.
\end{abstract}

Palabras clave: Ética médica; Mejoramiento de la calidad; Derechos humanos; Bioética (fuente: Decs BIREME).

\section{ETHICS AND MEDICINE: THE EXPERIENCE OF THE PERUVIAN MEDICAL ASSOCIATION}

\begin{abstract}
This article shows the work of the Peruvian Medical Association with respect to ethical disciplinary procedures, but also shows how, beyond the ethical control, the Order has promoted through a set of actions, a technically competent health care that respects the dignity and fundamental human rights. Part of these actions are the establishment of the Patient's Day, the emphasis in improving the quality and safety in the care of the users of the health services, the initiative to prevent and treat adverse events, the regulation and improvement of the physicians training both in pre as in postgraduate levels, and the demand for the establishment of an optimal health system and policies that will realize the right to health in the context of the principles of bioethics.
\end{abstract}

Key words: Ethics, medical; Quality improvement; Human rights; Bioethics (source: MeSH NHL).

\section{LA ÉTICA MÉDICA}

La Ética Médica, en una perspectiva más propiamente deontológica, alude al conjunto de normas de comportamiento que enmarcan el quehacer de los miembros de la profesión médica en sus relaciones con quienes demandan sus servicios y de aquellas que se establezcan entre si $^{(1)}$. Tal como señalábamos en un reciente artículo ${ }^{(2)}$ el impresionante desarrollo de la ciencia y de la tecnología médica, la tendencia a la superespecialización y la transformación de los hospitales en centros de alta tecnología, a menudo teñidos de un sesgo empresarial, han modificado radicalmente la relación médicopaciente y la relación entre la medicina y la sociedad.

También han contribuido a estos cambios la toma de conciencia por parte de los pacientes de su derecho a la salud; la introducción de las leyes del mercado y la aplicación acrítica del modelo industrial con énfasis inadecuado en criterios de productividad antes que de calidad de la atención.

\section{LAS FUNCIONES DEL COLEGIO MÉDICO DEL PERÚ}

\section{DE SUS FINES}

La Ley N. ${ }^{\circ} 15173$ del 16 de octubre de 1964, por la cual se crea el Colegio Médico del Perú (CMP) ${ }^{(3)}$, precisa en su Art. $5 .^{\circ}$, que constituyen fines de la Orden, entre otros, el velar para que el ejercicio profesional se cumpla de acuerdo con las normas deontológicas contenidas en el Código de Ética profesional que el Colegio dicte; propender a mejorar la salud individual y colectiva de los habitantes del país; contribuir al avance de la ciencia médica y cooperar

\footnotetext{
Comité de Vigilancia Ética y Deontológica, Consejo Nacional, Colegio Médico del Perú. Lima, Perú.

2 Instituto de Ética en Salud, Universidad Nacional Mayor de San Marcos. Lima, Perú.

a Médico Psiquiatra, Magister en Ciencias Familiares y Sexológicas
} 
con los poderes públicos en la defensa de la salud, procurando que la asistencia facultativa alcance a todo el país.

Desde que, en la práctica, el CMP iniciara su funcionamiento hacia 1969 , se ha esmerado en el cumplimiento de sus fines, contribuyendo al mejoramiento de la salud individual y colectiva a través de diversas acciones.

\section{DEL CÓDIGO DE ÉTICA Y DEONTOLOGÍA DEL COLEGIO MÉDICO DEL PERÚ}

El primer Código de Ética del Colegio data del año 1969, el que, recogiendo los principios y valores que prevalecían entonces, orientaba la conducta de los médicos hacia la procura del bienestar para los pacientes (principio de beneficencia), pero en el marco de una relación de corte paternalista, en la que el médico decidía lo que consideraba mejor para el paciente, sin que la opinión de este tuviera la relevancia que tiene hoy.

Los radicales cambios experimentados por la Medicina en las últimas décadas condujeron a la Orden a la actualización del Código primigenio. Así, el 5 de octubre del año 2000 , entró en vigencia un segundo Código ${ }^{(4)}$. Este fue, y se trata de un aspecto que merece resaltarse, el resultado de un amplio e intenso debate que se inició cuatro años antes de su aprobación y en el que participó no únicamente la profesión médica sino también la sociedad civil, a través de representantes de instituciones públicas y privadas relacionadas con la salud. Proceder de otro modo hubiera significado violentar la esencia misma de la ética que, siguiendo a Gracia ${ }^{(5)}$, debe ser civil o secular, pluralista, autónoma y racional.

Como fruto de este consenso se incorporaron los principios de la bioética, los derechos de los pacientes, la prohibición expresa de participar en actos de lesa humanidad, la defensa de la competencia ética del Colegio Médico sobre cualquier otra jurisdicción, y la responsabilidad social compartida del médico, la sociedad y el Estado para garantizar el derecho a la salud de todas las personas. Pero, como el impulso ético no puede quedarse fijado, una nueva revisión hizo posible la dación del actual Código que, aprobado el $2007^{(6)}$, entró en vigencia el año 2008. El nuevo Código desarrolla más ampliamente lo concerniente a los derechos de los pacientes y los conceptos de la bioética

\section{LA VIGILANCIA ÉTICA Y DEONTOLÓGICA}

\section{EL CONTROL ÉTICO DEONTOLÓGICO}

El CMP, a través de sus comités de vigilancia ética y deontológica (CVED), tanto del Consejo Nacional cuanto de sus veintisiete consejos regionales, tiene la obligación de difundir y vigilar el cumplimiento de la Ley, Estatuto, Reglamento, Código de Ética y Deontología y cualquier norma o acuerdo emanado del Consejo Nacional o de los Consejos Regionales en su caso. Si el CVED hallara indicios de transgresión a las normas antedichas, solicitará la apertura del procedimiento ético disciplinario, a cargo del Comité de Asuntos Contenciosos y Procedimientos Disciplinarios (CACYPD). El Reglamento indica el procedimiento a seguir en estos casos, el cual guarda similitud con lo establecido en los procesos judiciales, en salvaguarda de los derechos fundamentales del colegiado: presunción de inocencia, reserva del procedimiento, resolución absolutoria o sancionatoria fundamentada y derecho a la doble instancia.

En respuesta a estos problemas, el CMP ha venido desarrollando diversas actividades educativas, tanto en Lima como en las diversas regiones del país, a fin de fortalecer la vigilancia ética, apelando, en primer lugar, a la prevención, vía el mejoramiento de la calidad de la formación del médico y de su actualización permanente a través de los procesos de acreditación de facultades de medicina y de recertificación profesional (hoy obligatoria por mandato de la Ley del SINEACE), y, en segundo lugar, mediante seminarios, talleres y cursos en los que, de manera práctica e interactiva, se busca capacitar en el método de la deliberación, según el modelo de Gracia ${ }^{(7)}$ que permita, partiendo de lo que técnicamente es posible, deliberar sobre la mejor línea de acción deseable, articulando el conocimiento médico, es decir la competencia técnica, con la reflexión ética y las limitaciones y posibilidades dadas por la ley, todo lo cual contribuirá el mejoramiento de la calidad y la seguridad en la atención de las personas.

En el caso específico del crecimiento desmesurado de las facultades de medicina, el CMP alentó la creación y funcionamiento de la Comisión para la Acreditación de Facultades o Escuelas de Medicina (CAFME), en busca de la mejora de la calidad y la excelencia académica, tanto en el pre como en el posgrado, y fue el primer Colegio Profesional en instaurar el Sistema de Certificación y Recertificación Profesional (SISTCERE). Para darle sustento a sus propuestas el CMP propició el año 2007 una rigurosa investigación ${ }^{(8)}$ sobre el tema y presentó una iniciativa legislativa orientada a suspender temporalmente la creación de nuevas entidades formadoras, demanda que no se concretó por la debilidad de las instancias de gobierno encargadas de regular la formación de recursos humanos. 


\section{LOS PROCEDIMIENTOS ÉTICO DISCIPLINARIOS}

Entre los años 2000 y 2001, el CVED del Consejo Nacional examinó aproximadamente 90 denuncias y paralelamente hizo un seguimiento de 74 procesos que se ventilaban en el Poder Judicial y que involucraban a 156 médicos. Asimismo, en el año 2001, el Comité de Asuntos Contenciosos y Procedimientos Disciplinarios tramitó veintinueve casos, contra siete del año 2000. De ese total, diez fueron archivados, cuatro terminaron en multa para el denunciado, tres en amonestación privada, seis en amonestación pública, uno en proceso judicial y, por primera vez en la historia de la Orden, uno en expulsión.

Hace poco, Véliz ${ }^{(9)}$ realizó una investigación orientada a conocer las características de las denuncias contra médicos presentadas al Comité de Vigilancia Ética y Deontológica del Consejo Regional III - Lima. Con tal propósito seleccionó, por muestreo al azar, $148(10 \%)$ de un total de 1500 expedientes correspondientes al lapso entre 1980 y 1999 , trabajo que reveló una tendencia creciente de las denuncias.

El examen de las situaciones antecedentes, vale decir de aquellas que motivaron las denuncias, puso en evidencia que el $40 \%$ de estas estaba ligada con actos médicos y, por lo tanto, tenían que ver en gran medida, con la relación médico paciente; un $27 \%$ con problemas en los que habían intervenido los medios masivos de comunicación; un $16 \%$ giraba en torno a dificultades de relación interpersonal entre los miembros de la Orden y un $12 \%$ se asociaba con situaciones en las que el problema había llegado hasta el nivel policial o judicial.

Si lo vemos desde la perspectiva de quién hace la denuncia, apreciamos en la Tabla 1 que, cuando se trata de instituciones o autoridades médicas, el $55 \%$ de las denuncias -hechas por el Consejo Regional III o por los directores de hospitales- tenía que ver con transgresiones a los códigos o reglamentos, y el $31 \%$ con la relación médico-paciente, siendo el $9 \%$ pasibles de sanción penal y un porcentaje menor, $5 \%$, se relacionaba con transgresiones ligadas a factores económicos. Pero, cuando quienes denuncian son los pacientes o sus familiares, el $73 \%$ de las situaciones tiene que ver con la relación médico-paciente.

Si vemos estos problemas desde la perspectiva judicial, vale la pena reseñar los hallazgos de un estudio transversal, realizado entre mayo y junio de 1999, en los 37 Juzgados de Lima, el cual registró la existencia de 190 médicos procesados, 59 por homicidio culposo, 14 por lesiones culposas y 4 por exposición o abandono de personas en peligro, lo que representaba el 1,2\% de los médicos que por entonces ejercían en Lima, sin considerar los Juzgados del Ministerio Público, los de la región Callao y del Cono Norte. Este es un indicador valioso que nos revela que algunos médicos están siendo directamente denunciados al fuero judicial, siendo de suponer que en dichos casos haya un componente ético que sería necesario evaluar.

Volviendo a los casos del Consejo Regional III, cuando los denunciantes son los pacientes o sus familiares la mayor crítica se relaciona con culpa (daño no intencional) y luego con dolo (daño por la realización intencional de un acto ilícito), seguidos por falsificación de documentos o por transgresiones referidas a factores económicos. Cuando los denunciantes son autoridades médicas, instituciones $u$ otros médicos, las denuncias inciden en la transgresión de las normas administrativas que rigen el comportamiento de los médicos en los establecimientos de salud, principalmente públicos.

Entre los años 2004 - 2005, el Consejo Nacional examinó 51 casos, provenientes de los Comités correspondientes tanto de Lima como de las regiones. Del total de casos, 13 aproximadamente el $20 \%$, fueron absueltos; un número igual retornó a los Comités para su reevaluación a causa de sendos recursos de apelación, y 25 médicos fueron sancionados, seis de ellos con suspensión del ejercicio profesional (alguno por 15 días, otro por un año).

Tabla 1. Denuncias registradas en el Colegio Médico del Perú, 2004-2005.

\begin{tabular}{|c|c|c|}
\hline & $\mathbf{N}$ & $(\%)$ \\
\hline \multicolumn{3}{|c|}{ Por instituciones o autoridades médicas (54 denuncias) * } \\
\hline $\begin{array}{l}\text { Por transgresiones a } \\
\text { reglamentos o códigos de salud } \\
\text { (Incluye } 17 \text { por publicaciones } \\
\text { inadecuadas) }\end{array}$ & 32 & $(59,3)$ \\
\hline $\begin{array}{l}\text { Por transgresiones en relación } \\
\text { directa con pacientes (Incluye } 7 \\
\text { por intrusismo) }\end{array}$ & 18 & $(33,3)$ \\
\hline $\begin{array}{l}\text { Por transgresiones pasibles de } \\
\text { sanción penal }\end{array}$ & 5 & $(9,3)$ \\
\hline \multirow[t]{2}{*}{ Por transgresiones económicas } & 3 & $(5,5)$ \\
\hline & 54 & $(100,0)$ \\
\hline \multicolumn{3}{|l|}{$\begin{array}{l}\text { Por pacientes o sus familiares } \\
\text { (45 denunciantes, } 48 \text { denuncias) }\end{array}$} \\
\hline $\begin{array}{l}\text { Por transgresiones en relación } \\
\text { directa con pacientes }\end{array}$ & 35 & $(73,0)$ \\
\hline $\begin{array}{l}\text { Por transgresiones pasibles de } \\
\text { sanción penal }\end{array}$ & 6 & $(12,0)$ \\
\hline Por transgresiones económicas & 6 & $(12,5)$ \\
\hline \multirow[t]{2}{*}{ Sin datos } & 1 & $(2,0)$ \\
\hline & 48 & $(100,0)$ \\
\hline
\end{tabular}


En el periodo comprendido entre los años 20062007, el total de procedimientos ético disciplinarios evaluados por el Consejo Nacional llegó a 124 casos. De ellos, 59 (47,6 \%) fueron absueltos, disponiéndose el archivamiento del expediente; 36 (25\%), fueron apelados, y reenviados a los Comités; y 29 (22\%) recibieron sanciones, algunas muy severas, tales como amonestación pública y suspensión temporal del ejercicio profesional.

En todo el periodo, 61 (34,9\%) casos fueron vistos por el Comité de Vigilancia Ética Y Deontológica, y 114 (65,1\%) por el Comité de Asuntos Contenciosos y Procedimientos Disciplinarios. En la Tabla 2 se resumen las procedencias de las sanciones y sus tipos en ambos periodos.

Entre los años 2008 - 2009 el total de procedimientos ético disciplinarios llegó a 139, contra 124 del periodo precedente. La mayor parte de los casos provenían del Consejo Regional III, Lima, que llegó a 113 denuncias, mientras que el conjunto de casos procedentes de los consejos regionales llegó a 26.

Lo importante a reseñar es que 75 denuncias tenían que ver directamente con el acto médico, poniendo en evidencia la crisis en el establecimiento de una adecuada relación médico-paciente. Las quejas estaban

Tabla 2. Procedimientos ético disciplinarios elevados al Consejo Nacional en apelación, periodo 2004-2007.

\begin{tabular}{|c|c|c|c|}
\hline & $\begin{array}{l}2004- \\
2005\end{array}$ & $\begin{array}{l}2006- \\
2007\end{array}$ & TOTAL \\
\hline \multicolumn{4}{|l|}{ Los casos provienen de } \\
\hline Consejo Regional de Lima & 37 & 95 & 132 \\
\hline $\begin{array}{l}\text { Consejos Regionales a nivel } \\
\text { nacional }\end{array}$ & 13 & 26 & 39 \\
\hline $\begin{array}{l}\text { De oficio por el Consejo } \\
\text { Nacional }\end{array}$ & 1 & 3 & 4 \\
\hline \multicolumn{4}{|l|}{ Tipos de sanción } \\
\hline $\begin{array}{l}\text { Suspensión del ejercicio } \\
\text { profesional }\end{array}$ & 6 & 12 & 18 \\
\hline Amonestación Pública & 12 & 9 & 21 \\
\hline Amonestación Privada & 5 & 4 & 9 \\
\hline Nota de Extrañeza & 2 & 4 & 6 \\
\hline $\begin{array}{l}\text { Nueva revisión por } \\
\text { apelación }\end{array}$ & 13 & 36 & 49 \\
\hline Archivo & 13 & 59 & 72 \\
\hline \multicolumn{4}{|l|}{ Instancia de supervisión } \\
\hline $\begin{array}{l}\text { Comité de Vigilancia Ética y } \\
\text { Deontológica }\end{array}$ & 15 & 46 & 61 \\
\hline $\begin{array}{l}\text { Comité de Asuntos } \\
\text { Contenciosos y } \\
\text { Procedimientos } \\
\text { Disciplinarios }\end{array}$ & 36 & 78 & 114 \\
\hline
\end{tabular}

ligadas a supuesta negligencia médica, a la carencia de consentimiento informado $o$ al incumplimiento en la entrega de la historia clínica cuando era solicitada. En 47 casos las denuncias se referían a problemas de relaciones interpersonales entre los propios médicos, con acusaciones de auditorías médicas inadecuadas, abuso de autoridad o empleo de expresiones implicando falta de respeto, injuria o difamación que atentaban contra el honor personal. En trece casos, el propio CMP había iniciado denuncia de oficio ante casos de diversa índole (sobre todo presuntos actos de negligencia médica) que fueron difundidos por los medios radiales, televisivos o periodísticos. Finalmente, en cuatro casos las denuncias se referían a problemas tales como certificados de defunción, informes médicos y pericias médico-legales considerados inexactos o al otorgamiento indebido de descansos médicos, entre otras situaciones en las que algunas veces los propios establecimientos de salud podían verse cuestionados.

Existe la creencia de que los médicos se protegen entre sí y no sancionan. Los datos presentados revelan que no es así. También es verdad que muchas denuncias no tienen sustento y son archivadas, a pesar de lo cual es probable que ellas sigan incrementándose. Dos factores contribuyen a ello: primero, el propio desarrollo de la medicina que, al hacerse más intervencionista aumenta la posibilidad de accidentes exploratorios o terapéuticos $y$, segundo, el que los pacientes, fascinados por los avances tecnológicos, han desarrollado una expectativa casi mágica que los lleva a pensar que cualquier problema médico puede ser resuelto a entera satisfacción, lo que no siempre es posible, dado que en medicina (más que en cualquier otra actividad humana) se está siempre expuesto a lo imprevisible.

\section{EL COLEGIO MÉDICO DEL PERÚ Y LA BIOÉTICA: MÁS ALLÁ DE LA VIGILANCIA ÉTICA}

Por todo lo expuesto la regulación de la conducta profesional mediante la aplicación de los preceptos deontológicos es insuficiente. Debemos movernos del enfoque casuístico, centrado en la relación médicopaciente, hacia una ética de las organizaciones sanitarias, ampliando así nuestra mirada de los complejos problemas sanitarios desde una perspectiva sistémica. Mi tesis es que el CMP, recogiendo lo mejor de la tradición médica peruana, ha incidido en todos estos ámbitos.

Los derechos de los pacientes. En el plano de la ética personal, el CMP, reconociendo el derecho de toda persona a recibir una atención de calidad, introdujo en su Código, como ya lo hemos referido, un capítulo referen- 
te a los derechos de los pacientes, haciendo suya la Declaración de Huacachina de 1995, en la que los médicos de la Asociación Nacional de Médicos del Ministerio de Salud (ANMMS) los proclamaron dos años antes de que tales derechos fueran incorporados en la Ley General de Salud de 1997.

El consentimiento informado (principio de autonomía). El reconocimiento de la autodeterminación de los pacientes encuentra su expresión en la doctrina del consentimiento informado, cuya aplicación ha sido -y es- constantemente alentada por el CMP, junto con la recomendación de velar por un trato digno y respetuoso con el paciente, razón de ser de nuestra profesión.

La calidad y la seguridad en la atención del paciente (principios de beneficencia y no-maleficencia). El principio de beneficencia ha regido desde siempre el quehacer del médico. Si bien planteado en el Corpus Hippocraticum, el principio de no-maleficencia, inicialmente ligado al de beneficencia, ha ido adquiriendo cada vez mayor importancia en el mundo actual, al punto que, junto al principio de justicia, tiene un correlato jurídico, de manera tal que si el médico actuara de modo negligente, imprudente o imperito, y de ello resultara daño a la integridad del paciente, aquél tendrá que rendir cuenta no solo a la Orden en el plano ético, sino también al Poder Judicial y reparar (en lo posible) el daño causado.

El CMP, consciente de su papel en este campo, conformó el año 2008 el Comité Impulsor de la Campaña por la Calidad de la Atención y Seguridad del Paciente, orientado, entre otros objetivos, a Promover una cultura de seguridad y calidad de la atención en las organizaciones públicas y privadas de salud, y un trato digno a los usuarios y pacientes, en el marco del respeto a sus derechos y del ejercicio ético de la profesión médica.

El año 2010, el Consejo Nacional del CMP acordó que dicho comité se convirtiera en un Comité Asesor Permanente y, como parte de sus acciones, se buscó sensibilizar a las autoridades políticas en torno al tema, por lo que se suscribió un convenio con el Ministerio de Salud, al mismo tiempo que se desarrollaron diversas estrategias de capacitación a los profesionales de la salud, especialmente a los médicos, en lo referente a los aspectos éticos, legales y aquellos inherentes a la mejora continua de la calidad y la seguridad en la atención sanitaria, tales como cirugía segura, higiene de manos, trato digno al usuario y promoción del derecho a la salud.

Pero ello no fue todo; en la búsqueda de la disminución de riesgos en la atención de salud, el CMP haciendo uso de su derecho a iniciativa legislativa, propuso la creación del Programa de Prevención y Atención de Eventos Adversos en los establecimientos de salud (10), con la intención de minimizar los riesgos y garantizar el derecho de los pacientes a recibir de la propia institución la atención médico-quirúrgica que su situación requiriera y al otorgamiento de la ayuda social que le permita afrontar de modo inmediato los efectos del evento adverso, tanto en los servicios médicos públicos como en los privados.

El Colegio Médico del Perú y la salud pública (principio de justicia). Para Gostin (11) si la salud es un valor fundamental, entonces los valores de equidad y justicia social exigen una distribución justa de las condiciones necesarias para que las personas sean saludables, condiciones que incluyan una variedad de factores educativos, económicos, sociales y ambientales que son necesarios para una buena salud.

En esta línea, el CMP se ha pronunciado desde un inicio por el establecimiento de un sistema de salud integrado, descentralizado, participativo y financiado; por la universalización de la seguridad social y el aseguramiento en salud; por el acceso universal a los medicamentos esenciales; por la mejora de la salud pública, privilegiando a los sectores secularmente excluidos y cuya pobreza los hace más vulnerables a todo tipo de enfermedades; por el respeto a los derechos de los pueblos amazónicos y otros grupos étnicos que conforman nuestro país, y cuyos ecosistemas deben ser preservados, estableciéndose con ellos un diálogo alturado y respetuoso con miras a lograr soluciones concordadas, entre otros temas que forman parte de la agenda sanitaria ${ }^{(12)}$ del CMP y que encuentran una sólida fundamentación en los principios de la bioética y los derechos fundamentales de la persona.

\section{LA FORMACIÓN EN BIOÉTICA Y LA CAPACITACIÓN DE LOS MÉDICOS EN TEMAS ÉTICOS Y DEONTOLÓGICOS}

El CMP, entre los años 1998 - 1999, promovió la formación de médicos en Bioética, contando con el valioso apoyo de la Organización Panamericana de Salud (OPS) y de la Universidad Nacional Mayor de San Marcos (UNMSM). El año 2000, la UNMSM inició el Diplomado en Bioética, el cual dio origen entre los años 2003 y 2004 a la Maestría Internacional de Bioética, dirigida por el profesor Diego Gracia, en un esfuerzo conjunto de la Universidad Complutense de Madrid, la UNMSM, la OPS y el CMP. Con el mismo propósito, el CMP ha venido realizando diversas actividades, principalmente en provincias, a fin de promover la capacitación en el campo ético y deontológico. En la última década se ha llegado a todas las regiones del país, habiéndose efectuado 
más de doscientos cursillos, seminarios y talleres con participación de renombrados eticistas peruanos y extranjeros.

Zavala ${ }^{(13)}$ en su trabajo “¿Para qué sirve la ética?”, nos muestra claramente cómo el Código de Ética puede ser de gran utilidad en el análisis de casos y la toma de decisiones adecuadas en la práctica cotidiana. En los últimos años la capacitación en aspectos ético legales se ha conjugado con aquellos orientados al mejoramiento continuo de la calidad y la seguridad del paciente, incidiendo en la ética de las organizaciones sanitarias.

\section{EL DÍA DEL PACIENTE}

El 22 de diciembre de 2010, el Ministerio de Salud, mediante Resolución Ministerial N. 1009, instituyó el 13 de agosto de cada año como el "Día del Paciente en el Perú".

Fue el Decano actual de la Orden, Dr. Ciro Maguiña, el gestor de la idea, la cual fue aprobada por el Consejo Nacional en marzo de 2010. A petición del Decano Nacional tuve el honor de preparar la Exposición de Motivos correspondiente, para el Proyecto de Ley que fue elevado al Congreso Nacional. Transcribo parte del párrafo final, el que puede leerse como las conclusiones a todo lo expuesto ${ }^{(14)}$.

Frente a ello, y como parte del movimiento en favor de la humanización de la atención de salud, que se expresa en un trato digno y la oferta de una asistencia sanitaria segura y de calidad, el CMP propone el establecimiento del Día del Paciente, el 13 de agosto, como un día destinado a suscitar una profunda reflexión tanto en cuidadores como en pacientes sobre el valor de la vida y por extensión de la salud, y sobre el significado de estar enfermos y las responsabilidades que en el cuidado de la salud competen al paciente, los profesionales de la salud, la sociedad y el Estado. Ese día se organizará en todos los establecimientos de salud del país una serie de actividades asistenciales, tanto de promoción de la salud cuanto de prevención de la enfermedad, asi como jornadas orientadas a proveer información en torno a los derechos de los pacientes, y a deliberar sobre el modo cómo cuidadores y pacientes pueden cambiar el sistema de salud insertándolo en el marco de una atención segura y de calidad y respetuosa de los derechos humanos, retomando el camino señalado por Daniel A. Carrión, paradigma de filantropía, abnegación y sacrificio.

\section{REFERENCIAS BIBLIOGRÁFICAS}

1. Lolas, F. Bioética. Santiago de Chile: Editorial Universitaria; 1998.

2. Mendoza A. Fundamentación de la enseñanza de la ética médica y la bioética clínica. Acta méd peruana. 2009;26(2):131-34.

3. Colegio Médico del Perú. Ley de Creación y Modificación. Estatuto. Reglamento. Código de Ética y Deontología. Trabajo Médico, Ley y Resolución. Ley General de Salud. Lima: Consejo Nacional del CMP; 2008.

4. Colegio Médico del Perú. Código de Ética y Deontología. Lima: Consejo Nacional del CMP; 2000.

5. Gracia D. Planteamiento general de la bioética. En: Couceiro A (editora). Bioética para clínicos. Madrid: ed. Triacastela; 1999. p. 19-35.

6. Colegio Médico del Perú. Código de Ética y Deontología. Lima: Consejo Nacional del CMP; 2007

7. Gracia D. La deliberación moral. El papel de las metodologías en ética. En: Asociación de Bioética Fundamental y Clínica (editores). Jornada de Debate sobre Comités Asistenciales de Ética. Madrid: Dirección General del Instituto Nacional de Salud; 1999. p. 21-41.

8. Carrasco V, Lozano E, Velásquez E. Análisis actual y prospectivo de la oferta y demanda de médicos en el Perú 2005-2011. Acta méd peruana. 2008;25(1):22-9.

9. Véliz J. Características de 148 denuncias presentadas al Comité de Vigilancia Ética y Deontológica del Consejo Regional III del Colegio Médico del Perú. Acta méd peruana. 2000;20(1):40-7.

10. Castro J, Carrasco V (editores). Memoria Gestión 2008-2009. Lima: CMP; 2010.

11. Gostin L. Teorías de la justicia y responsabilidad social en salud pública. En: Málaga $\mathrm{H}$, editor. Salud Pública. Enfoque Bioético. Caracas: editorial Disinlimed CA; 2005. p. 21-32.

12. Colegio Médico del Perú. Aportes del CMP a la Salud Pública del país. En: Castro J, Carrasco V (editores). Memoria Gestión 2008 - 2009. Lima: CMP; 2010. p. 3044.

13. Zavala S. ¿Para qué sirve la ética? Acta méd peruana. 2008;25(1):48-9.

14. Mendoza A. Día del Paciente. Acta méd peruana. 2010;27(2):144-7.

Correspondencia: Alfonso R. Mendoza Fernández Dirección: Las Palmeras 305 - Dpto. 901, Lima 27, Perú Teléfono: (511) 4420139 / 999098291

Correo electrónico: alfonsomendozaf@yahoo.com 